\title{
LYAPUNOV EXPONENTS AND OTHER PROPERTIES OF $N$-GROUPS
}

\author{
D. A. FILIMONOV AND V. A. KLEPTSYN
}

\begin{abstract}
We study the class of minimally acting finitely generated groups of $C^{2}$ diffeomorphisms of the circle which have the property that the nonexpandable points are fixed, where the set of nonexpandable points is nonempty. It turns out that the Lyapunov expansion exponent of any such action is zero. As a consequence, we have a singularity of the stationary measure for a random dynamical system given by any probability distribution whose support is a finite set of the generating elements of the group.
\end{abstract}

\section{INTRODUCTION}

In [5] and [3], in connection with the well-known conjecture on the ergodicity of minimal smooth actions of finitely generated groups on the circle, the following definition was given.

Definition 1. A point $x \in S^{1}$ is said to be nonexpandable (for the action of the group $G)$ if

$$
\forall g \in G \quad\left|g^{\prime}(x)\right| \leq 1
$$

The existence of such points is an obstacle to realizing Sullivan's technique of exponential expansion; we denote the set of nonexpandable points by $\mathrm{NE}=\mathrm{NE}(G)$.

Definition 2. A finitely generated group $G \subset \operatorname{Diff}^{2}\left(S^{1}\right)$ is called an $N$-group if its action is minimal, $\mathrm{NE}(G) \neq \varnothing$, and for each point $x \in \mathrm{NE}(G)$ there exist $g_{+}, g_{-} \in G$ such that $g_{+}(x)=g_{-}(x)=x$ and $x$ is an isolated point from the right (from the left) of $\operatorname{Fix}\left(g_{+}\right)$ $\left(\operatorname{Fix}\left(g_{-}\right)\right.$, respectively).

Note that the presence of nonexpandable points does not contradict the minimality of the action of the group of (even analytic) diffeomorphisms: examples include the standard action of $\mathrm{PSL}_{2}(\mathbb{Z})$ and (for the smooth case) the smooth realization by GhysSergiescu [7] of the Thompson group. However, all the examples of minimal actions with nonexpandable points which are currently known differ from these two only in insignificant details; in particular, they are all $N$-groups.

The main aim of this paper is to study $N$-groups, in particular the corresponding Lyapunov exponents and the consequences for stationary measures. For simplicity we shall confine ourselves to orientation-preserving diffeomorphisms, since such diffeomorphisms form a subgroup of index at most 2, and passing to a subgroup of finite index preserves minimality.

Previously, in 5 it was shown that the action of an $N$-group is ergodic with respect to Lebesgue measure and that the set of nonexpandable points for any such group is finite.

2010 Mathematics Subject Classification. Primary 37C85; Secondary 37E10, 37A35, 37D25, 37H15.

Key words and phrases. Dynamical systems, group actions, diffeomorphisms of the circle, Lyapunov exponent, stationary measures.

This work was carried out with the partial support of the Russo-French programme "Cooperation network in mathematics", grant RFFI-10-01-00739-a and grant RFFI-CNRS-10-01-93115-NTsNIL-a. 
Then a theorem was proved in 3 ] describing the action of such a group; we recall this theorem in $\S 2$ below.

To formulate our main result, Theorem 1 below, we recall the following definition.

Definition 3. Let $G \subset \operatorname{Diff}^{2}\left(S^{1}\right)$ be a finitely generated group, and let $\mathcal{F}=\mathcal{F}^{-1}$ be a finite symmetric system of its generators. Then

$$
\lambda_{\exp }(x ; \mathcal{F})=\limsup _{n \rightarrow \infty} \frac{1}{n} \max _{f_{1}, \ldots, f_{n} \in \mathcal{F}} \ln \left|\left(f_{1} \circ \ldots \circ f_{n}\right)^{\prime}(x)\right|
$$

is called the Lyapunov expansion exponent at the point $x$.

Note that Hurder [10] proved that, for a minimal action of a finitely generated group $G \subset \operatorname{Diff}^{2}\left(S^{1}\right)$, the function $\lambda_{\exp }(\cdot, \mathcal{F})$ coincides almost everywhere (with respect to Lebesgue measure) with some constant $\lambda_{\exp }(G, \mathcal{F})$, called the Lyapunov expansion exponent of the group $G$. Although the size of this constant depends on the choice of the system of generators, the question of whether it is zero or positive does not.

Our main result is the following theorem, which asserts that exponential growth for an $N$-group is impossible.

Theorem 1. The Lyapunov expansion exponent of an $N$-group $G$ is zero:

$$
\lambda_{\exp }(G)=0 .
$$

On the other hand, for groups with zero expansion exponent, the stationary measures for finitely generated dynamics are singular. Thus we have the following corollary.

Corollary 1. Let $G$ be an $N$-group, and let $m$ be a probability measure on it whose support consists of finitely many elements and which, as a subgroup, generates $G$. Then the (unique) m-stationary measure is singular with respect to Lebesgue measure.

A similar assertion for the action of the group $\operatorname{PSL}(2, \mathbb{Z})$ was announced by Givarch and Le Jan [8] and proved by Givarch and Raja [9], Proposition 15.

\section{Preliminaries}

Here we give two theorems from [3] describing the action of an $N$-group. The first asserts the existence of a "Markov" partition and an almost-everywhere expanding dynamical system.

Theorem 2 (the Markov partition, [3]). Let $G$ be a finitely generated $N$-group of $C^{2}$ diffeomorphisms of the circle. Then for $G$ there exists a partition of the circle into intervals

$$
\left\{I_{1}, \ldots, I_{k}, I_{1}^{+}, I_{1}^{-}, \ldots, I_{l}^{+}, I_{l}^{-}\right\}=\mathcal{J}
$$

and maps corresponding to these intervals:

$$
g_{1}, \ldots, g_{k}, g_{1}^{+}, g_{1}^{-}, \ldots, g_{l}^{+}, g_{l}^{-} \in G
$$

such that:

i) all the images $g_{i}^{ \pm}\left(I_{i}^{ \pm}\right), g_{j}\left(I_{j}\right)$ are represented as unions of intervals in $\mathcal{J}$;

ii) $\exists \lambda>1: \forall j \forall x \in I_{j} g_{j}^{\prime}(x) \geqslant \lambda$;

iii) the intervals $I_{i}^{+}$and $I_{i}^{-}$are respectively right-and left-adjacent to a nonexpandable point $x_{i}^{*} \in \mathrm{NE}$. This is a fixed topologically repelling point of the restriction of the map $g_{i}^{+}\left(g_{i}^{-}\right)$to the interval $I_{i}^{+}\left(I_{i}^{-}\right.$, respectively), which has no other fixed points on this interval.

Furthermore,

$$
\forall i \forall x \in I_{i}^{ \pm} \quad\left(g_{j(x)} \circ\left(g_{i}^{ \pm}\right)^{k_{i}^{ \pm}(x)}\right)^{\prime}(x) \geqslant \lambda,
$$


where $k_{i}^{ \pm}(x):=\min \left\{k \in \mathbb{N}:\left(g_{i}^{ \pm}\right)^{k}(x) \notin I_{i}^{ \pm}\right\}$, and $j(x)$ is defined by the condition $\left(g_{i}^{ \pm}\right)^{k_{i}^{ \pm}(x)}(x) \in I_{j(x)}$;

iv) Finally, all the boundary points of the partition belong to the orbits of nonexpandable points.

We fix a partition $\mathcal{J}$ from the conclusion of this theorem; furthermore, for convenience, we denote the map corresponding to an interval $I$ in the chosen partition map by $g_{I}$. By grouping the maps $g_{I}$ into a piecewise continuous map

$$
R: S^{1} \rightarrow S^{1},\left.\quad R\right|_{I}=g_{I} \quad \forall I \in \mathcal{J},
$$

we can use iterations of this map as a kind of (local) "microscope" enabling us to expand the separate intervals (in particular, this idea was used in the proof of ergodicity in [5]).

The second of the theorems in [3] asserts that under this magnification all the maps in $G$ turn out to be constructed from finitely many identical elementary "building blocks".

Theorem 3 (the structure of the action, [3]). There exist finitely many intervals $L_{1}, \ldots$, $L_{N}, L_{1}^{\prime}, \ldots, L_{N}^{\prime} \subset S^{1}$ and maps $h_{i}: L_{i} \rightarrow L_{i}^{\prime}$ such that any map $g \in G$ can be represented in the following way:

- there are two partitions (depending on the choice of $g$ ) of the circle into a union of intervals:

$$
S^{1}=J_{1} \cup \ldots \cup J_{m}=g\left(J_{1}\right) \cup \ldots \cup g\left(J_{m}\right) ;
$$

- for each $p=1, \ldots, m$ there exist $n, n^{\prime}, i_{p}$, such that

$$
R^{n}\left(J_{p}\right)=L_{i_{p}}, \quad R^{n^{\prime}}\left(g\left(J_{p}\right)\right)=L_{i_{p}}^{\prime},
$$

where $R^{n}$ and $R^{n^{\prime}}$ are continuous on the intervals $J_{p}$ and $g\left(J_{p}\right)$, repectively, and under this "magnification" the map g turns out to be the map $h_{i_{p}}$ :

$$
\left.g\right|_{J_{p}}=\left.\left(\left.R^{n^{\prime}}\right|_{g\left(J_{p}\right)}\right)^{-1} \circ h_{i_{p}} \circ R^{n}\right|_{J_{p}} .
$$

Furthermore, the partition $S^{1}=\bigcup J_{i}$ can be chosen to be the same for any finite collection of maps in $G$.

In other words, by construction, the action of the group $G$ is reminiscent of the GhysSergiescu smooth action of the Thompson group [7].

Furthermore, as is readily seen from the proof in [3], all the intervals $L_{i}$ are in fact Markov; that is, they belong to $\mathcal{J}$, although some of them may be equal and some of the elements of $\mathcal{J}$ may be absent from the $\left\{L_{i}\right\}$. In what follows we shall call a local representation

$$
\left.g\right|_{J}=\left.\left(\left.R^{n^{\prime}}\right|_{g(J)}\right)^{-1} \circ h \circ R^{n}\right|_{J},
$$

where $R^{n}(J) \in\left\{L_{i}\right\}_{i=1}^{m} \subset \mathcal{J}$, a representation in the sense of Theorem 3 .

\section{Proof of the MAIN RESUlt}

In a certain sense, the iterations of $R$ give rise to a "greedy" algorithm for expanding a neighbourhood of a given point. We therefore begin by establishing that, in view of the presence of parabolic points, applying such an algorithm does not enable us to achieve an exponential rate of expansion.

Theorem 4. For almost any point $x \in S^{1}$ (with respect to Lebesgue measure) the Lyapunov exponent of the map $R$ at this point is equal to zero:

$$
\lambda(x, R)=0 .
$$


The proof in fact repeats the proof of Theorem B in [5]. Namely, we note that the prelimit expression $\frac{1}{n} \ln \left(R^{n}\right)^{\prime}(x)$ can be written as

$$
\frac{1}{n} \ln \left(R^{n}\right)^{\prime}(x)=\frac{1}{n} \sum_{j=0}^{n-1} \Phi\left(R^{j}(x)\right)=\int_{S^{1}} \Phi(y) d \mu_{n, x}(y),
$$

where $\Phi(y)=\ln R^{\prime}(y)$ and $\mu_{n, x}$ is the time average of the point $x$ :

$$
\mu_{n, x}=\frac{1}{n} \sum_{j=0}^{n-1} \delta_{R^{j}(x)} .
$$

Since the map $R$ is piecewise $C^{1}$-smooth and both of its one-sided derivatives at any nonexpandable point are equal to 1 (and, accordingly, the limit of the piecewise continuous function $\Phi$ at such points exists and is equal to zero), it suffices to show that the following holds.

Lemma 1. For almost any point $x \in S^{1}$, the measures $\mu_{n, x}$ converge to the set of atomic measures $\left\{\sum_{y \in N E} \alpha_{y} \delta_{y}\right\}$ concentrated on $\mathrm{NE}$; in other words, for any neighbourhood $U$ of the set $\mathrm{NE}$, we have

$$
\mu_{n, x}\left(S^{1} \backslash U\right) \underset{n \rightarrow \infty}{\longrightarrow} 0 .
$$

This lemma can be derived from the existence of an infinite absolutely continuous $R$ invariant measure for Markov maps with parabolic fixed points, as worked out in Bowen's paper [1, and our arguments (taking the first-return map on the part bounded away from the parabolic points and the existence of a smooth finite invariant measure on it) are to a large degree similar to those used there. However, for the sake of completeness, we shall give a proof.

Proof of the lemma. We denote the union of the Markov intervals adjacent to the nonexpandable points by $U_{0}:=\bigcup_{j} I_{j}^{ \pm}$. In the original construction of Theorem 2 , by choosing a preliminary partition of the circle that is sufficiently fine (see [3]), we can assume that the following holds for the union $U_{0}$ : if $x \in I_{j}^{ \pm}$and $g_{j}^{ \pm}(x) \notin I_{j}^{ \pm}$, then $g_{j}^{ \pm}(x) \notin U_{0}$. In other words, after one iteration of the expansion procedure, one cannot leave one of the intervals adjacent to $\mathrm{NE}$ and pass immediately into another.

We now consider the map $\widetilde{R}$ of the first $R$-return to $S^{1} \backslash U_{0}$ and the return-time function $T$ :

$$
T(x):=\min \left\{k>0: R^{k}(x) \in S^{1} \backslash U_{0}\right\}, \quad \widetilde{R}(x):=R^{T(x)}(x) .
$$

The assertion of the lemma then follows from an estimate of the behaviour of the time averages of the return time (which we prove below):

Proposition 1. For almost any point $x \in S^{1} \backslash U_{0}$ (with respect to Lebesgue measure),

$$
\lim _{k \rightarrow \infty} \frac{1}{k} \sum_{j=0}^{k-1} T\left(\widetilde{R}^{j}(x)\right)=+\infty .
$$

Indeed, suppose that the proposition has been proved. Note that a typical point $x \in S^{1}$ (with respect to Lebesgue measure) after finitely many iterations of $R$ becomes a typical point $x \in S^{1} \backslash U_{0}$ (with respect to Lebesgue measure). Therefore, without loss of generality, we can assume from the very beginning that we are working with points $x \in S^{1} \backslash U_{0}$.

For any point $x \in S^{1} \backslash U_{0}$ for which (3.1) holds, we shall divide its sequence of $R$ iterations into segments until it returns to $S^{1} \backslash U_{0}$ : 


$$
\ldots, \underbrace{\widetilde{R}^{k}(x), R\left(\widetilde{R}^{k}(x)\right), \ldots, R^{T\left(\widetilde{R}^{k}(x)\right)-1}\left(\widetilde{R}^{k}(x)\right)}_{\text {segment number } k}, \widetilde{R}^{k+1}(x), \ldots
$$

If a neighbourhood $U$ of the set NE is chosen and fixed, then on each such segment a point will be outside $U$ at most a constant $C_{U}$ number of times (depending on $U$ ); the time until departure from $U_{0}$ for points in $S^{1} \backslash U$ (detached from NE) is uniformly bounded above. Accordingly, after $N$ iterations, where

$$
T(x)+T(\widetilde{R}(x))+\ldots+T\left(\widetilde{R}^{k-1}(x)\right) \leq N<T(x)+T(\widetilde{R}(x))+\ldots+T\left(\widetilde{R}^{k}(x)\right),
$$

there cannot be more than $C_{U} \cdot(k+1)$ of them outside $U$ and the fraction of them is estimated as

$$
\mu_{N, x}\left(S^{1} \backslash U\right) \leq \frac{C_{U} \cdot(k+1)}{N} \leq C_{U} \cdot \frac{k+1}{\sum_{j=0}^{k-1} T\left(\widetilde{R}^{j}(x)\right)} .
$$

In view of (3.1), the ratio on the right-hand side tends to zero.

Thus, the proof of Lemma 1 (and, along with it, Theorem 4) will be completed as soon as Proposition 1 is proved.

Proof of Proposition 1. Note that (similar to the corresponding derivation in [11] and [5], Lemma 5.5) the map $\widetilde{R}$ consists of (countably many) branches, the image of each of which completely covers the union of several Markov intervals. Moreover, all of these branches expand with a derivative bounded away from 1 and uniformly bounded by the distortion norm (see [5]).

Therefore (similar to the arguments in [12]; see also [1]), for $\widetilde{R}$-iterations of Lebesgue measure, the logarithm of the density on each interval of $\mathcal{J}$ turns out to be uniformly Lipschitzian.

Hence (as follows from the Krylov-Bogolyubov procedure), $\widetilde{R}$ has an absolutely continuous invariant measure, where the logarithm of its density is Lipschitz on each Markov interval (and possibly the density vanishes identically on some intervals).

Next, since the map $\widetilde{R}$ is expanding, such a measure $\mu$ is either ergodic or is a linear combination of finitely many ergodic components, each of which is the restriction of $\mu$ to some union of Markov intervals that preserves $\widetilde{R}$.

On the other hand, the function $T: S^{1} \backslash U_{0} \rightarrow \mathbb{N}$ of the return time in a neighbourhood of any point that converts into a nonexpandable point under the action of $R$ has a singularity that is of the form $1 / x$ or worse. Furthermore, by construction, each boundary point of the Markov interval goes over to a nonexpandable point after a finite number of $R$-iterations; therefore, the function $T$ will have such a singularity either in this neighbourhood or in one of its $\widetilde{R}$-images. Finally, a singularity of the form $1 / x$ for the function $T$ and the absolute continuity with nonzero density of the measure $\mu$ means that the following integral diverges:

$$
\int_{S^{1} \backslash U_{0}} T d \mu=+\infty
$$

Furthermore, the same is true for each of the ergodic components of $\mu$.

Therefore, by the Birkhoff-Khinchin theorem, for almost any $x$ (with respect to the measure $\mu$ ) and, consequently, with respect to Lebesgue measure, we have

$$
\frac{1}{k} \sum_{j=0}^{k-1} T\left(\widetilde{R}^{j}(x)\right) \rightarrow+\infty, \quad k \rightarrow \infty
$$

For the proof of Theorem 1 we give the following definition. 
Definition 4. By the height of a representation of a map $g \in G$ in the sense of Theorem 3 ,

$$
\left.g\right|_{J}=\left.\left(\left.R^{n}\right|_{g(J)}\right)^{-1} \circ h_{j} \circ R^{m}\right|_{J}
$$

we mean the number $m$, and by the depth, we mean the number $n$.

Note that the technique of Theorem 3 can be applied to the maps $h_{j}$ themselves by reducing to their furthest subdivision:

$$
\begin{aligned}
&\left.h_{i}\right|_{U_{i, j}}=\left.\left(\left.R^{n_{i, j}}\right|_{h_{i}\left(U_{i, j}\right)}\right)^{-1} \circ h_{l_{i, j}} \circ R^{m_{i, j}}\right|_{U_{i, j}}, \\
& m_{i, j}>0, \quad R^{m_{i, j}}\left(U_{i, j}\right) \in\left\{L_{k}\right\} \subset \mathcal{J}, \quad \bigcup_{j} U_{i, j}=L_{i} .
\end{aligned}
$$

This implies the following result.

Proposition 2. Let $\mathcal{F} \subset G$ be a fixed finite set. Then there is an $M>0$ such that if $\tilde{g} \in \mathcal{F}$ and in a neighbourhood of some point $x$ the map $g$ admits a representation in the sense of Theorem 3 of height $m$, then the composite $\tilde{g} \circ g$ in a (possibly smaller) neighbourhood of the same point admits a representation of height at most $m+M$.

Similarly, there is a constant $M^{\prime}$ such that if $g$ admits a representation of depth $n$, then $g \circ \tilde{g}$ admits a representation of depth at most $m+M^{\prime}$.

Proof. We choose and fix some representations of all the maps in $\mathcal{F}$ and let $M_{1}$ be the maximum of the heights of all the chosen representations, $M_{2}:=\max _{i . j} m_{i, j}$.

Then by repeating (if necessary) the subdivision procedure for the corresponding maps $h_{j}$ in a neighbourhood of the point $g(x)$, we can find a representation for $\tilde{g}$ of height $m^{\prime}$ with $n \leq m^{\prime} \leq n+\max \left(M_{1}, M_{2}\right)$.

Thus, in the corresponding neighbourhood $U$ of the point $x$,

$$
\begin{gathered}
\tilde{g} \circ g=\left.\left.\left(\left.R^{n^{\prime}}\right|_{\tilde{g} \circ g(U)}\right)^{-1} \circ h_{i} \circ R^{m^{\prime}}\right|_{g(U)} \circ\left(\left.R^{n}\right|_{g(U)}\right)^{-1} \circ h_{j} \circ R^{m}\right|_{U} \\
=\left.\left(\left.R^{n^{\prime}}\right|_{\tilde{g} \circ g(U)}\right)^{-1} \circ\left(h_{i} \circ R^{m^{\prime}-n} \circ h_{j}\right) \circ R^{m}\right|_{U} .
\end{gathered}
$$

Finally, the composites $h_{i} \circ R^{k} \circ h_{j}$ with $0 \leq k \leq \max \left(M_{1}, M_{2}\right)$ form a finite collection of maps. We denote by $M$ the largest of the heights of their representations, and by substituting this representation into (3.3), we obtain the required result.

The second conclusion of the proposition is proved completely analogously.

Proposition 3. Let $\mathcal{F} \subset G$ be a fixed finite set. Then there is a constant $C_{0}>0$ such that for any $g_{1}, \ldots, g_{s} \in \mathcal{F}$ and for any point in some neighbourhood $U$ of it, the composite $g_{1} \circ \cdots \circ g_{s}$ admits a representation in the sense of Theorem 3:

$$
\left.g\right|_{U}=\left.\left(\left.R^{n}\right|_{g(U)}\right)^{-1} \circ h_{j} \circ R^{m}\right|_{U}
$$

for which neither the height nor the depth exceeds $C_{0} \cdot s$.

Proof. Applying Proposition 2 to the composite $g=g_{1} \circ\left(g_{2} \circ\left(g_{3} \circ \ldots \circ g_{s}\right)\right)$, we see that in some neighbourhood $J$ of $x$ we have a representation in the sense of Theorem 3 :

$$
\left.g\right|_{J}=\left.\left(\left.R^{n}\right|_{g(J)}\right)^{-1} \circ h_{i} \circ R^{m}\right|_{J}, \quad R^{m}(J)=L_{i} \in \mathcal{J},
$$

of height $m \leq M s$. On the other hand, it follows from $g=\left(\left(g_{1} \circ g_{2}\right) \circ \cdots \circ g_{s}\right)$ that in some neighbourhood $J^{\prime}$ of $x$ we have the representation

$$
\left.g\right|_{J^{\prime}}=\left.\left(\left.R^{n^{\prime}}\right|_{g\left(J^{\prime}\right)}\right)^{-1} \circ h_{j} \circ R^{m^{\prime}}\right|_{J^{\prime}}, \quad R^{m^{\prime}}\left(J^{\prime}\right)=L_{j} \in \mathcal{J},
$$

of depth $n^{\prime} \leq M^{\prime} s$.

If $n \leq n^{\prime}$ or $m \leq m^{\prime}$, then (3.4) or (3.5) satisfies the condition of the lemma. Suppose now that $n>n^{\prime}$ and $m>m^{\prime}$. Then, in view of the Markov property, conditions 
$R^{m}(J) \in \mathcal{J}, R^{m^{\prime}}\left(J^{\prime}\right)=R^{m^{\prime}-m}\left(R^{m}\left(J^{\prime}\right)\right) \in \mathcal{J}$, and the presence of the point $x \in J \cap J^{\prime}$, we have $R^{m}\left(J^{\prime}\right) \subset R^{m}(J)$ and therefore $J^{\prime} \subset J$.

By rewriting the restriction to $J^{\prime}$ and the representations (3.4) and (3.5) in the form

$$
\left.h_{i} \circ R^{m}\right|_{J^{\prime}}=\left.R^{n} \circ g\right|_{J^{\prime}}=\left.R^{n-n^{\prime}} \circ\left(R^{n^{\prime}} \circ g\right)\right|_{J^{\prime}}=\left.R^{n-n^{\prime}} \circ\left(h_{j} \circ R^{m^{\prime}}\right)\right|_{J^{\prime}},
$$

we get

In particular,

$$
\left.h_{i}\right|_{R^{m}\left(J^{\prime}\right)}=\left.R^{n-n^{\prime}} \circ h_{j} \circ R^{m^{\prime}-m}\right|_{R^{m}\left(J^{\prime}\right)} .
$$

But the length of the interval on the right-hand side of (3.6) is nonzero, since the map $h_{j}$ (chosen from a finite set) and a positive power of $R$ are successively applied to the interval $L_{j}=R^{m^{\prime}}\left(J^{\prime}\right) \in \mathcal{J}$, which has nonzero length. Applying $h_{i}^{-1}$ to the left-hand side, we find that the length of $R^{m}\left(J^{\prime}\right)$ is nonzero, but through $m^{\prime}-m$ iterations, this interval becomes a Markov interval $L_{j} \in \mathcal{J}$. Taking into account that the map $R$ is expanding, we see that there is a uniform upper bound on $m^{\prime}-m$.

Thus in this case too we can use the representation (3.5) - its depth is at most $M^{\prime} s$ and its height $m^{\prime}$ is estimated as the sum $m \leq M s$ and does not exceed the constant for the quantity $m^{\prime}-m$. This completes the proof of Proposition 3.

We now prove Theorem 1 .

Proof. We fix a symmetric system $\mathcal{F}$ of generators of the group $G$ and apply the preceding Proposition 3 to it. Then for any point $x$, any composite $g=f_{1} \circ \ldots \circ f_{s}$ of the maps $f_{1}, \ldots, f_{s} \in \mathcal{F}$ in a neighbourhood $U$ of this point can be represented as

$$
g=\left.\left(\left.R^{n}\right|_{g(U)}\right)^{-1} \circ h_{i} \circ R^{m}\right|_{U},
$$

where $m \leq M \cdot s$. Hence

$$
g^{\prime}(x)=\frac{h_{i}^{\prime}\left(R^{m}(x)\right) \cdot\left(R^{m}\right)^{\prime}(x)}{\left(R^{n}\right)^{\prime}(g(x))} \leq \frac{C \cdot \max _{1 \leq j \leq M s}\left(R^{j}\right)^{\prime}(x)}{\left(R^{n}\right)^{\prime}(g(x))}
$$

where $C:=\max _{x \in S^{1}} \max _{i \leq N} h_{i}^{\prime}(x)$.

Therefore,

$$
\frac{1}{s} \ln g^{\prime}(x) \leq \frac{\ln C}{s}+\frac{1}{s} \cdot \max _{1 \leq j \leq M s} \ln \left(R^{j}\right)^{\prime}(x)-\frac{1}{s} \ln \left(R^{n}\right)^{\prime}(g(x)) .
$$

The first term clearly tends to 0 ; that the second term converges to 0 for almost any point $x \in S^{1}$ follows from Theorem 4. It remains to find an upper bound for the third term.

Note that when $G$ is in fact a group of piecewise analytic diffeomorphisms, the strict inequality $R^{\prime}>1$ holds in a neighbourhood of any nonexpandable point; therefore, the derivatives of $R^{n}$ are uniformly bounded below and the conclusion of Theorem 1 now follows immediately.

We now consider the general case.

Note that, in view of the definition of the map $R$, the derivative of its iteration is at least 1 at any moment when the point leaves the neighbourhood of the nonexpandable points: $R^{k}(x) \in S^{1} \backslash U_{0} \Rightarrow\left(R^{k}\right)^{\prime}(x)>1$. Therefore, the derivative $\left(R^{n}\right)^{\prime}(g(x))$ splits up into a product of derivatives $\left(R^{k_{j}}\right)^{\prime}\left(R^{k_{1}+\cdots+k_{j-1}}(g(x))\right)>1$, where $R^{k_{1}}(g(x)), \ldots$, $R^{k_{1}+\cdots+k_{t}}(g(x)) \in S^{1} \backslash U_{0}$, and the remainder

$$
\left(R^{n-\left(k_{1}+\ldots+k_{t}\right)}\right)^{\prime}\left(R^{k_{1}+\ldots+k_{t}}(g(x))\right)=\prod_{i=k_{1}+\ldots+k_{t}}^{n-1} R^{\prime}\left(R^{i}(g(x))\right)
$$


where

$$
\forall i, k_{1}+\ldots+k_{t} \leq i<n, \quad R^{i}(g(x)) \in U_{0} .
$$

It remains for us to find a lower bound for the expression

$$
\frac{1}{s} \ln \left(R^{n-\left(k_{1}+\ldots+k_{t}\right)}\right)^{\prime}\left(R^{k_{1}+\ldots+k_{t}}(g(x))\right)=\frac{n}{s} \cdot \frac{1}{n} \sum_{i=k_{1}+\ldots+k_{t}}^{n-1} \ln R^{\prime}\left(R^{i}(g(x))\right) .
$$

But, in view of the condition (3.7), outside any $\varepsilon$-neighbourhood of the set NE there exists at most a constant $C(\varepsilon)$ number of points $R^{i}(g(x))$ in the iterates under consideration. On the other hand, $\ln R^{\prime}(y) \rightarrow 0$ as $y \rightarrow \mathrm{NE}$ and, finally, $n / s \leq C_{0}$.

Hence it is easy to see that (3.8) is estimated by a quantity that tends to zero as $s \rightarrow \infty$. This completes the proof of the theorem.

\section{ACKNOWLEDGEMENTS}

The authors would like to thank E. Ghys, Yu. S. Il'yashenko, D. V. Anosov, B. Deroin, A. Navas and I. G. Khovanskaya for valuable discussions and their interest in this work.

\section{REFERENCES}

[1] R. Bowen, Invariant measures for Markov maps on the interval, Comm. Math. Phys. 69 (1979), 1-17. MR.547523 (81e:28010)

[2] A. B. Katok and B. Hasselblatt, Introduction to the theory of dynamical systems with a survey of latest achievements, Cambridge University Press, Cambridge, 2006.

[3] V. A. Kleptsyn and D. A. Filimonov, On actions on the circle with the fixed-point property for nonexpandable points, Funktsional. Anal. i Prilozhen, to appear.

[4] M. Herman, Sur la conjugaison différentiable des difféomorphismes du circle à des rotations, Publ. Math. Inst. Hautes Études Sci. 49 (1979), 5-234. MR538680 (81h:58039)

[5] B. Deroin, V. Kleptsyn and A. Navas, On the question of ergodicity for minimal group actions on the circle, Moscow Math. J. 9 (2009), no. 2, 263-303. MR2568439 (2010m:37041)

[6] B. Deroin, V. Kleptsyn and A. Navas, Sur la dynamique unidimensionelle en régularité intermédiaire, Acta Math. 199 (2007), no. 2, 199-262. MR2358052 (2010c:37059)

[7] É. Ghys and V. Sergiescu, Sur un groupe remarquable de difféomorphismes du cercle, Comment. Math. Helv. 62 (1987), 185-239. MR896095 (90c:57035)

[8] Y. Guivarc'h and Y. Le Jan, Asymptotic winding of the geodesic flow on modular surfaces and continuous fractions, Ann. Sci. École Norm. Sup. (4) 26 (1993), no. 1, 23-50. MR1209912(94a:58157)

[9] Y. Guivarc'h and C. R. E. Raja, Recurrence and ergodicity of random walks on linear groups and on homogeneous spaces, Preprint arXiv:0908.0637.

[10] S. Hurder, Exceptional minimal sets and the Godbillon-Vey class, Ann. Inst. Fourier (Grenoble), to appear.

[11] T. Inoue, Ratio ergodic theorems for maps with indifferent fixed points, Ergodic Theory Dynam. Systems 17 (1997), 625-642. MR1452184(98e:58109)

[12] M. Mañé, Introducäo à teoria ergódica, Instituto de Matemática Pura e Aplicada (1983). MR800092 (87d:58085)

Moscow State University

E-mail address: mityafil@gmail.com

CNRS, Institut de Recherche Mathématique de Rennes

E-mail address: victor.kleptsyn@univ-rennes1.fr 